\title{
NUMERICAL STUDY OF THREE-PARAMETER MATRIX EIGENVALUE PROBLEM BY GRADIENT METHOD
}

\author{
Niranjan Bora ${ }^{1 \S}$, Arun Kumar Baruah ${ }^{2}$ \\ ${ }^{1,2}$ Department of Mathematics, DUIET \\ Dibrugarh University \\ Dibrugarh, 786004, Assam, INDIA
}

\begin{abstract}
In this paper, a Convergent Gradient Method, which was first developed to solve generalised eigenvalue problem of the form $A x=\lambda B x$, will be used to find approximate eigenvalues and corresponding eigenvectors of Multiparameter Matrix Eigenvalue Problems (MMEPs). To apply Gradient Method, we will reduce MMEPs into a single matrix pencil containing the block diagonal matrices. Although the Method can be extended to Multiparameter case, but the whole work is done by considering three-parameter case only to relax computational cost. Computational efficiency of this method will be discussed by comparing the results with Kronecker Product Method as proposed by Atkinson in 1960, analytically with the help of numerical examples.
\end{abstract}

AMS Subject Classification: 35PXX, 65FXX, 65F15, 35A35

Key Words: multiparameter matrix eigenvalue problems, Kroneecker product, tensor product space, convergent gradient method

\section{Introduction}

The MMEPs are the more generalization concept of classical one-parameter standard eigenvalue problems of the form $A x=\lambda x$ and the generalized eigenvalue problems of the form $A x=\lambda B x$, where the problem is to find a k-tuple of values $\lambda=\left(\lambda_{1}, \lambda_{2}, \ldots \lambda_{k}\right) \in C^{k}$ and non-zero vector $x_{i} \in C^{n_{i}}$ for $i=1,2 \ldots k$

Received: $\quad$ February 25, 2016

Revised: $\quad$ October 24, 2016

Published: January 24, 2017

$\S_{\text {Correspondence author }}$ (c) 2017 Academic Publications, Ltd. url: www.acadpubl.eu 
such that

$$
\left(A_{i}-\Sigma_{j=1}^{k} \lambda_{j} B_{i j}\right) x_{i}=0, \quad i=1,2, \ldots k,
$$

where $\lambda_{i} \in R^{i} ; i=1,2,3, \ldots k$ are spectral parameters and $A_{i}, B_{i j} ; j=$ $1,2,3 \ldots k$ are self-adjoint, bounded linear operators that act in Hilbert Spaces $H_{i}, x_{i} \in H_{i}$. The k-tuple $\lambda \in C^{k}$ is called an eigenvalue and the tensor product $x=x_{1} \otimes x_{2} \otimes x_{3} \otimes \ldots \otimes x_{k}$ is the corresponding (right) eigenvector. Similarly left eigenvector can also be defined. This type of problems arises in various physical situations, particularly where the separation of variables technique is used to solve a discretised boundary value problem. In $1960 \mathrm{~s}$, mainly due to Atkinson's works [[4], [5]], a new stage began in the investigation of MMEPs and he has introduced an abstract algebraic setting (1) from the viewpoint of determinantal operators on the tensor product space. He showed that a nonsingular MEP is equivalent to the associated system of generalized eigenvalue problems $A x=\lambda B x$. Many theoretical results as well as numerical methods for non-singular MMEPs are based on this relation.

Numerical solution of MMEPs for matrices arises in the discretization of Multiparameter Sturm-Liouville eigenvalue problems in ordinary differential equations. Only a few numerical methods are available for such a problems, particularly for two-parameter case. For example continuation method [2], JacobiDavidson's method [6], Bisection Method [9] etc. The connection between stability of invariant subspace of algebraic MMEPs and the stability of invariant subspaces of commuting matrices are found in [8], which is very important for numerical calculation.

The problem defined by the equation (1) is relatively new one in numerical analysis. Its solution is very complicated due to the lack of spectral theory for the case $k \geq 2$ as comparable to the theory for $k=1$. Atkinson showed that the separation of spectral parameters is possible directly for the case $k=2$ in tensor product space, which can not be done for the case $k \geq 3$. There is no standard method for treating such problem for $k \geq 3$ in the existing literature. In this paper an attempt will be done to study three-parameter matrix eigenvalue problems numerically with the help of Gradient Method presented in [7] to enrich the Multiparameter cases.

The rest of the paper is organized as follows: Section 2 contains some basic preliminaries. In Section 3 a brief description of three-parameter problem and in section 4 a convergent Gradient Method is presented. Similarly in Section 5 the efficiency of the method is tested with the help of numerical examples and finally in section 6 a conclusion and the future scope of study is drawn. 


\section{Basic Preliminaries}

Definition 1. F be a real functional defined on the Hilbert space $\mathrm{H}$. The frechet derivative of $\mathrm{F}$ at $x \in H$ is a linear operator, denoted by $\Delta F(x)$ such that

$$
\lim _{h \rightarrow 0} \frac{\|F(x+h)-F(x)-\Delta F(h)\|}{\|h\|}=0 .
$$

For $\mathrm{H}$ with real inner product $\langle x, y\rangle$ we can write the linear operator $\Delta F(h)=$ $\langle\Delta F(x), h\rangle$

Definition 2. A point $x \in H$ is called stationary point of $\mathrm{F}$ if $\Delta F(x)=0$. A subset $E \subset H$ is called stationary set of $\mathrm{F}$ if $\Delta F(x)=0, \forall x \in E$.

\section{Three-Parameter Matrix Eigenvalue Problem and its Abstract Settings by Atkinson}

Three-parameter matrix problem is a particular case of (1) for $i=3$

$$
\begin{aligned}
& \left(A_{1}-\lambda_{1} B_{11}-\lambda_{2} B_{12}-\lambda_{3} B_{13}\right) x_{1}=0, \\
& \left(A_{2}-\lambda_{1} B_{21}-\lambda_{2} B_{22}-\lambda_{3} B_{23}\right) x_{2}=0, \\
& \left(A_{3}-\lambda_{1} B_{31}-\lambda_{2} B_{32}-\lambda_{3} B_{33}\right) x_{3}=0 .
\end{aligned}
$$

An eigenvalue is defined to be a 3 -tuple $\left(\lambda_{1}, \lambda_{2}, \lambda_{3}\right)$ of complex numbers for which there exists a non-zero decomposable element $u=x_{1} \otimes x_{2} \otimes x_{3}$. The necessary definiteness condition is found in [4] and is given by

$$
D\left(u_{1}, u_{2}, u_{3}\right)=\left|\begin{array}{lll}
\left(B_{11} u_{1}, u_{1}\right) & \left(B_{12} u_{1}, u_{1}\right) & \left(B_{13} u_{1}, u_{1}\right) \\
\left(B_{21} u_{2}, u_{2}\right) & \left(B_{22} u_{2}, u_{2}\right) & \left(B_{23} u_{2}, u_{2}\right) \\
\left(B_{31} u_{3}, u_{3}\right) & \left(B_{32} u_{3}, u_{3}\right) & \left(B_{33} u_{3}, u_{3}\right)
\end{array}\right| \geq 0,
$$

where $\left(B_{i j} u_{i}, u_{i}\right)$ are the auxilary inner product. Problem (2) is called right definite if conditions (3) are satisfied and can be reduced into the three generalized one parameter problems in the tensor product space given by

$$
\begin{aligned}
\Delta_{1} u & =\lambda_{1} \Delta_{0} u, \Delta_{2} u=\lambda_{2} \Delta_{0} u, \Delta_{3} u=\lambda_{3} \Delta_{0} u, \\
\Delta_{0}^{-1} \Delta_{1} u & =\lambda_{1} u, \Delta_{0}^{-1} \Delta_{2} u=\lambda_{2} u, \Delta_{0}^{-1} \Delta_{3} u=\lambda_{3} u,
\end{aligned}
$$

where:

$$
\Delta_{0}=\left|\begin{array}{ccc}
\tilde{B_{11}} & \tilde{B_{12}} & \tilde{B_{13}} \\
\tilde{B_{21}} & \tilde{B_{22}} & \tilde{B_{23}} \\
\tilde{B_{31}} & \tilde{B_{32}} & \tilde{B_{33}}
\end{array}\right|, \quad \Delta_{1}=\left|\begin{array}{ccc}
\tilde{A_{1}} & \tilde{B_{12}} & \tilde{B_{13}} \\
\tilde{A_{2}} & \tilde{B_{22}} & \tilde{B_{23}} \\
\tilde{A_{3}} & \tilde{B_{32}} & \tilde{B_{33}}
\end{array}\right|
$$




$$
\Delta_{2}=\left|\begin{array}{ccc}
\tilde{B_{11}} & \tilde{A_{1}} & \tilde{B_{13}} \\
\tilde{B_{21}} & \tilde{A_{2}} & \tilde{B_{23}} \\
\tilde{B_{31}} & \tilde{A_{3}} & \tilde{B_{33}}
\end{array}\right|, \quad \Delta_{3}=\left|\begin{array}{ccc}
\tilde{B_{11}} & \tilde{B_{12}} & \tilde{A_{1}} \\
\tilde{B_{21}} & \tilde{B_{22}} & \tilde{A_{2}} \\
\tilde{B_{31}} & \tilde{B_{32}} & \tilde{A_{3}}
\end{array}\right|
$$

and

$$
u=x_{1} \otimes x_{2} \otimes x_{3},
$$

where the induced operators $\tilde{A}_{i}$ and $\tilde{B}_{i j}$ in tensor product space are the operators induced by $A_{i}$ and $B_{i j}$ respectively in $H_{i}, i=1,2,3$. The induced operators are defined by $\tilde{A_{1}}=A_{1} \otimes I_{2} \otimes I_{3}, \tilde{A_{2}}=I_{1} \otimes A_{2} \otimes I_{3}, \tilde{A_{3}}=I_{1} \otimes I_{2} \otimes A_{3}, \tilde{B_{1 i}}=$ $B_{1 i} \otimes I_{2} \otimes I_{3}, \tilde{B_{2 i}}=I_{1} \otimes B_{2 i} \otimes I_{3}$ and $\tilde{B_{3 i}}=I_{1} \otimes I_{2} \otimes B_{3 i}$ where $I_{i}, i=1,2,3$ are the identity operators in $H_{i}, i=1,2,3$. MMEP is right definite $\Leftrightarrow \Delta_{0}$ is nonsingular. The operators $G_{i}=\Delta_{0}^{-1} \Delta_{i}, i=1,2,3$ commute. If MMEP is right definite then

1. Eigen 3-tuple are real.

2. There exist $n^{3}$ linearly independent eigenvectors.

3. Eigenvectors of distinct eigenvalues are $\Delta_{0}$-orthogonal, i.e., $\left(x_{1} \otimes x_{2} \otimes\right.$ $\left.x_{3}\right)^{T} \Delta_{0}\left(x_{1} \otimes x_{2} \otimes x_{3}\right)=0$.

\section{The Gradient Method for Matrix Pencils}

The method was first developed to solve generalized eigenvalue problem of the form $A x=\lambda B x$ [Presented in [3]] and later generalized to solve Matrix Eigenvector-Eigentuple Problems of the form $A x=\sum_{i=1}^{i=k} \lambda_{i} B_{i} x$, [presented in [1], [7] by Blum etc.]. First we consider the matrix pencil of the form

$$
\left(A-\sum_{i=1}^{i=k} \lambda_{i} B_{i}\right) x=0
$$

where $A, B_{i}$ are linear operators in Hilbert Spaces $H$. A vector $x \in H$, $x \neq 0$ satisfying (5) for a given $\lambda=\left(\lambda_{1}, \lambda_{2}, \ldots \lambda_{k}\right)$ is called eigenvector of the Matrix Eigenvector-Eigentuple Problems. Let for each eigenvector $\mathrm{x}$ the set of vectors $\left\{B_{1} x, B_{2} x \ldots, B_{k} x\right\}$ is linearly independent and this assumption is essential to remove the degenerate case. Define $\alpha_{i}(x)=\left\langle A x, B_{i} x\right\rangle$, $\alpha(x)=\left(\alpha_{1}(x), \alpha_{2}(x), \ldots, \alpha_{k}(x)\right), \beta_{i j}(x)=\left\langle B_{i} x, B_{j} x\right\rangle$ and

$$
\beta(x)=\left(\beta_{i j}(x)\right),
$$


where $1 \leq i, j \leq k$. Let $\alpha(x)$ be the column vector with elements $\alpha_{i}(x)$ and Let $\beta^{-1}(x)$ exist. Let $\wedge(x)$ be defined by the equation

$$
\wedge(x)=\beta^{-1}(x) \alpha(x) .
$$

Consider the functional

$$
F(x)=\frac{1}{2}\left\|A x-\sum_{i=1}^{i=n} \lambda_{i} B_{i} x\right\|^{2},
$$

defined for all $x \in H$ such that $\beta^{-1}(x)$ exists. And $\lambda_{i}$ are the components of the vector $\wedge(x)$ in (7). It can be proved that the eigenvectors of (1) are the stationary points of $\mathrm{F}$ and conversely. We define

$$
\Delta F(x)=\left(A-\sum_{i=1}^{i=n} \lambda_{i} B_{i}\right)^{*}\left(A-\sum_{i=1}^{i=n} \lambda_{i} B_{i}\right) x .
$$

We now define the Gradient Method from [3]

$$
h(x)= \begin{cases}-2 F(x) \Delta F(x) /\|\Delta F(x)\|^{2} & \text { if } \Delta F(x) \neq 0, \\ 0 & \text { if } \Delta F(x)=0,\end{cases}
$$

and

$$
x_{k+1}=x_{k}+h\left(x_{k}\right), \quad k=0,1,2, \ldots .
$$

To find the solution of (5) we have to choose initial gauss $x_{0}$ on the unit sphere and then compute $\wedge\left(x_{0}\right), F(x)$ and $\Delta F(x)$ from the equations (7), (8) and (9) respectively. Then after calculating $h\left(x_{0}\right)$, the next iteration $x_{1}$ will be determined using the equations (10) and (11). Since in general $x_{1}$ may not be on the unit shpere and hence by normalizing $x_{1}$ the procedure will be repeated. The iteration (11) will be continuied in this way such that $x_{m}$ and its corresponding k-tuple $\wedge\left(x_{m}\right)$ is the approximate solution of the problem (5). This iterative procedure can be used to solve three-parameter eigenvalue problems (1) by reducing the same into a single matrix pencil, which is discussed in the following section.

\subsection{Conversion of Three-Parameter Matrix Eigenvalue Problem into a Single Matrix Pencil}

The system (2) can be written in the following form 


$$
\begin{aligned}
& \left(\begin{array}{ccc}
A_{1} & 0 & 0 \\
0 & A_{2} & 0 \\
0 & 0 & A_{3}
\end{array}\right)\left(\begin{array}{ccc}
x_{1} & 0 & 0 \\
0 & x_{2} & 0 \\
0 & 0 & x_{3}
\end{array}\right)=\left\{\lambda_{1}\left(\begin{array}{ccc}
B_{11} & 0 & 0 \\
0 & B_{21} & 0 \\
0 & 0 & B_{31}
\end{array}\right)\right. \\
& \left.+\lambda_{2}\left(\begin{array}{ccc}
B_{12} & 0 & 0 \\
0 & B_{22} & 0 \\
0 & 0 & B_{32}
\end{array}\right)+\lambda_{2}\left(\begin{array}{ccc}
B_{13} & 0 & 0 \\
0 & B_{23} & 0 \\
0 & 0 & B_{33}
\end{array}\right)\right\}\left(\begin{array}{ccc}
x_{1} & 0 & 0 \\
0 & x_{2} & 0 \\
0 & 0 & x_{3}
\end{array}\right) . \\
& \Rightarrow A X=\left(\lambda_{1} B_{1}+\lambda_{2} B_{2}+\lambda_{3} B_{3}\right) X,
\end{aligned}
$$

where:

$$
A=\left(\begin{array}{ccc}
A_{1} & 0 & 0 \\
0 & A_{2} & 0 \\
0 & 0 & A_{3}
\end{array}\right), \quad B_{i}=\left(\begin{array}{ccc}
B_{1 i} & 0 & 0 \\
0 & B_{2 i} & 0 \\
0 & 0 & B_{3 i}
\end{array}\right), \quad X=\left(\begin{array}{ccc}
x_{1} & 0 & 0 \\
0 & x_{2} & 0 \\
0 & 0 & x_{3}
\end{array}\right) .
$$

Obviously the problem formulation of the equation (2) and that of the equation (12) are equivalent. Although, Gradient Method discussed in the Section 3 can't be applied directly to find a common solution of the system (2), but this conversion into single matrix pencil allow us to deal with the problem successfully.

Theorem 1. Let $R: R^{n} \rightarrow R$ be the function defined by $F(x)=$ $\frac{1}{2}\left\|\left(A-\Sigma \lambda_{i} B_{i}\right) x\right\|^{2}$ where $A, B_{i}$ are $n \times n$ matrix. Then the Frechet derivative of $F$ at $x_{0}$ is a function $M$, where

$$
M(h)=x_{0}^{T}\left(A-\Sigma \lambda_{i} B_{i}\right)^{T}\left(A-\Sigma \lambda_{i} B_{i}\right) h
$$

Proof. We have

$$
\begin{aligned}
F\left(x_{0}+h\right)= & \frac{1}{2}\left\|\left(A-\Sigma \lambda_{i} B_{i}\right)\left(x_{0}+h\right)\right\|^{2} \\
= & \frac{1}{2}\left\|\left(A-\Sigma \lambda_{i} B_{i}\right) x_{0}+\left(A-\Sigma \lambda_{i} B_{i}\right) h\right\|^{2} \\
= & \frac{1}{2}\left\{\left\|\left(A-\Sigma \lambda_{i} B_{i}\right) x_{0}\right\|^{2}+\|\left(A-\Sigma \lambda_{i} B_{i}\right)\right) h \|^{2} \\
& \left.+2\left[\left(A-\Sigma \lambda_{i} B_{i}\right) x_{0}\right]^{T}\left(A-\Sigma \lambda_{i} B_{i}\right) h\right\} \\
= & \frac{1}{2}\left\|\left(A-\Sigma \lambda_{i} B_{i}\right) x_{0}\right\|^{2}+\frac{1}{2}\left\|\left(A-\Sigma \lambda_{i} B_{i}\right) h\right\|^{2} \\
& +x_{0}^{T}\left(A-\Sigma \lambda_{i} B_{i}\right)^{T}\left(A-\Sigma \lambda_{i} B_{i}\right) h \\
= & F\left(x_{0}\right)+M(h)+\frac{1}{2}\left\|\left(A-\Sigma \lambda_{i} B_{i}\right) h\right\|^{2} .
\end{aligned}
$$


Hence it follows the theorem. Thus, the Frechet derivative of $\mathrm{F}$ at $x_{0}$ is given by

$$
\Delta F\left(x_{0}\right)=x_{0}^{T}\left(A-\Sigma \lambda_{i} B_{i}\right)^{T}\left(A-\Sigma \lambda_{i} B_{i}\right),
$$

which is useful at the calculation part of the equation (9).

Theorem 2. If $\Delta F(x)=0$ then $F(x)=0$.

Proof: See Lemma 3.1 pp. 252 - 253 [1].

\subsection{Finding of Stationary Point by B-R Gradient Procedure}

Let $x_{0}$ be the initial point in $H$. Let $\lambda_{i}\left(x_{0}\right)$ be the component of $\wedge\left(x_{0}\right)$. Define $x_{n}$ for $n \geq 1$ recursively by

$$
y_{n+1}=x_{n}+h\left(x_{n}\right), \quad x_{n+1}=\frac{y_{n+1}}{\left\|y_{n+1}\right\|},
$$

where

$$
h\left(x_{n}\right)= \begin{cases}\frac{-2 F\left(x_{n}\right) \Delta F\left(x_{n}\right)}{\left\|\Delta F\left(x_{n}\right)\right\|^{2}} & \text { if } \Delta F(x) \neq 0, \\ 0 & \text { otherwise. }\end{cases}
$$

The sequence $x_{n}$ converges to a stationary point of $\mathrm{F}$.

\subsection{Validity Conditions}

To apply Gradient Method for MMEPs, it is essential to remove degeneracy case of (12). To deal with the degeneracy case, we need the assumption that the set $\left\{B_{1} X, B_{2} X, B_{3} X\right\}$ is linearly independent.

Theorem 3. The set $\left\{B_{1} X, B_{2} X, B_{3} X\right\}$ is linearly independent if the sets $\left\{B_{11} x_{1}, B_{12} x_{1}, B_{13} x_{1}\right\} ;\left\{B_{21} x_{2}, B_{22} x_{2}, B_{23} x_{2}\right\}$ and $\left\{B_{31} x_{3}, B_{32} x_{3}, B_{33} x_{3}\right\}$ are linearly independent.

Proof. Assume that

$$
\left\{B_{11} x_{1}, B_{12} x_{1}, B_{13} x_{1}\right\}, \quad\left\{B_{21} x_{2}, B_{22} x_{2}, B_{23} x_{2}\right\}
$$

and

$$
\left\{B_{31} x_{3}, B_{32} x_{3}, B_{33} x_{3}\right\}
$$

are linearly independent.

Therefore

$$
\alpha_{1} B_{11} x_{1}+\alpha_{2} B_{12} x_{1}+\alpha_{3} B_{13} x_{1}=0,
$$




$$
\alpha_{1} B_{21} x_{2}+\alpha_{2} B_{22} x_{2}+\alpha_{3} B_{23} x_{2}=0
$$

and

$$
\begin{aligned}
& \alpha_{1} B_{31} x_{3}+\alpha_{2} B_{32} x_{3}+\alpha_{3} B_{33} x_{3}=0 \Rightarrow \alpha_{i}=0, \quad \forall i=1,2,3 . \\
& \Rightarrow \alpha_{1}\left(\begin{array}{ccc}
B_{11} x_{1} & 0 & 0 \\
0 & B_{21} x_{2} & 0 \\
0 & 0 & B_{31} x_{3}
\end{array}\right)+\alpha_{2}\left(\begin{array}{ccc}
B_{12} x_{1} & 0 & 0 \\
0 & B_{22} x_{2} & 0 \\
0 & 0 & B_{32} x_{3}
\end{array}\right) \\
&+\alpha_{3}\left(\begin{array}{ccc}
B_{13} x_{1} & 0 & 0 \\
0 & B_{23} x_{2} & 0 \\
0 & 0 & B_{33} x_{3}
\end{array}\right)=0,
\end{aligned}
$$

$\forall i=1,2,3$.

If $\alpha_{i}=0$ :

$$
\Rightarrow \alpha_{1} B_{1} X+\alpha_{2} B_{2} X+\alpha_{3} B_{3} X=0, \quad \forall i=1,2,3 .
$$

$\alpha_{i}=0$ :

$\Rightarrow\left\{B_{1} X, B_{2} X, B_{3} X\right\}$ is linearly independent.

Hence Gradient Method can be applied to three-parameter eigenvalue problems if $\left\{B_{11} x_{1}, B_{12} x_{1}, B_{13} x_{1}\right\} ;\left\{B_{21} x_{2}, B_{22} x_{2}, B_{23} x_{2}\right\}$ and $\left\{B_{31} x_{3}, B_{32} x_{3}, B_{33} x_{3}\right\}$ are linearly independent. Again from equation (6) we have

$$
\begin{gathered}
\beta(x)=\left(\begin{array}{lll}
\left\langle B_{1} X, B_{1} X\right\rangle & \left\langle B_{1} X, B_{2} X\right\rangle & \left\langle B_{1} X, B_{3} X\right\rangle \\
\left\langle B_{2} X, B_{1} X\right\rangle & \left\langle B_{2} X, B_{2} X\right\rangle & \left\langle B_{2} X, B_{3} X\right\rangle \\
\left\langle B_{3} X, B_{1} X\right\rangle & \left\langle B_{3} X, B_{2} X\right\rangle & \left\langle B_{3} X, B_{3} X\right\rangle
\end{array}\right), \\
\beta(x)=\left(\begin{array}{lll}
\operatorname{Tr}\left[\left(B_{1} X\right)^{T} B_{1} X\right] & \operatorname{Tr}\left[\left(B_{2} X\right)^{T} B_{1} X\right] & \operatorname{Tr}\left[\left(B_{3} X\right)^{T} B_{1} X\right] \\
\operatorname{Tr}\left[\left(B_{1} X\right)^{T} B_{2} X\right] & \operatorname{Tr}\left[\left(B_{2} X\right)^{T} B_{2} X\right] & \operatorname{Tr}\left[\left(B_{3} X\right)^{T} B_{2} X\right] \\
\operatorname{Tr}\left[\left(B_{1} X\right)^{T} B_{3} X\right] & \operatorname{Tr}\left[\left(B_{2} X\right)^{T} B_{3} X\right] & \operatorname{Tr}\left[\left(B_{3} X\right)^{T} B_{3} X\right]
\end{array}\right), \\
\beta(x)= \\
\left(\begin{array}{llll}
\operatorname{Tr}\left(X^{T} B_{1}^{T} B_{1} X\right) & \operatorname{Tr}\left(X^{T} B_{2}^{T} B_{1} X\right) & \operatorname{Tr}\left(X^{T} B_{3}^{T} B_{1} X\right) \\
\operatorname{Tr}\left(X^{T} B_{1}^{T} B_{2} X\right) & \operatorname{Tr}\left(X^{T} B_{2}^{T} B_{2} X\right) & \operatorname{Tr}\left(X^{T} B_{3}^{T} B_{2} X\right) \\
\operatorname{Tr}\left(X^{T} B_{1}^{T} B_{3} X\right) & \operatorname{Tr}\left(X^{T} B_{2}^{T} B_{3} X\right) & \operatorname{Tr}\left(X^{T} B_{3}^{T} B_{3} X\right)
\end{array}\right), \\
\left.\begin{array}{llll}
\operatorname{Tr}\left(B_{1}^{T} B_{1}\right) & \operatorname{Tr}\left(B_{2}^{T} B_{1}\right) & \operatorname{Tr}\left(B_{3}^{T} B_{1}\right) \\
\operatorname{Tr}\left(B_{1}^{T} B_{2}\right) & \operatorname{Tr}\left(B_{2}^{T} B_{2}\right) & \operatorname{Tr}\left(B_{3}^{T} B_{2}\right) \\
\operatorname{Tr}\left(B_{1}^{T} B_{3}\right) & \operatorname{Tr}\left(B_{2}^{T} B_{3}\right) & \operatorname{Tr}\left(B_{3}^{T} B_{3}\right)
\end{array}\right)
\end{gathered}
$$

Gradient Method can be applied for three-parameter matrix eigenvalue problem if $\beta^{-1}(x)$ exist i.e when $|\beta(x)| \neq 0$. Since all $B_{i}$ of the matrix $\beta(x)$ above are known from equation $(2)$ and hence $|\beta(x)|$ can be calculated directly to check validity. 


\begin{tabular}{|l|l|l|}
\hline Sl No & $\left(\lambda_{3}, \lambda_{3}, \lambda_{3}\right)$ & Eigen Vectors \\
\hline 1 & $(-0.0231,+0.0136,+0.2069)$ & $(100000000000000000000000000)^{T}$ \\
\hline 2 & $(-0.0246,+0.0135,+0.2112)$ & $(010000000000000000000000000)^{T}$ \\
\hline 3 & $(-0.0408,+0.0130,+0.2576)$ & $(001000000000000000000000000)^{T}$ \\
\hline 4 & $(-0.0234,+0.0045,+0.2092)$ & $(000100000000000000000000000)^{T}$ \\
\hline 5 & $(-0.0247,+0.0043,+0.2128)$ & $(000010000000000000000000000)^{T}$ \\
\hline 6 & $(-0.0418,+0.0015,+0.2621)$ & $(000001000000000000000000000)^{T}$ \\
\hline 7 & $(-0.0230,+0.0164,+0.2061)$ & $(000000100000000000000000000)^{T}$ \\
\hline 8 & $(-0.0246,+0.0160,+0.2108)$ & $(000000010000000000000000000)^{T}$ \\
\hline 9 & $(-0.0409,+0.0118,+0.2580)$ & $(000000001000000000000000000)^{T}$ \\
\hline 10 & $(+0.1345,+0.0014,+0.1968)$ & $(000000000100000000000000000)^{T}$ \\
\hline 11 & $(+0.1426,+0.0015,+0.1784)$ & $(000000000010000000000000000)^{T}$ \\
\hline 12 & $(+0.01083,+0.0011,+0.2557)$ & $(000000000001000000000000000)^{T}$ \\
\hline 13 & $(+0.1351,-0.0038,+0.1980)$ & $(000000000000100000000000000)^{T}$ \\
\hline 14 & $(+0.1433,+0.0028,+0.1789)$ & $(000000000000010000000000000)^{T}$ \\
\hline 15 & $(+0.1087,-0.0070,+0.2589)$ & $(000000000000001000000000000)^{T}$ \\
\hline 16 & $(+0.1340,+0.0056,+0.1958)$ & $(000000000000000100000000000)^{T}$ \\
\hline 17 & $(+0.1417,+0.0071,+0.1776)$ & $(000000000000000010000000000)^{T}$ \\
\hline 18 & $(+0.1083,+0.0006,+0.2559)$ & $(000000000000000001000000000)^{T}$ \\
\hline 19 & $(+0.2838,-0.0102,+0.1872)$ & $(000000000000000000100000000)^{T}$ \\
\hline 20 & $(+0.2872,-0.0090,+0.1876)$ & $(000000000000000000010000000)^{T}$ \\
\hline 21 & $(+0.2777,-0.0124,+0.2536)$ & $(000000000000000000001000000)^{T}$ \\
\hline 22 & $(+0.2841,-0.0116,+0.0358)$ & $(000000000000000000000100000)^{T}$ \\
\hline 23 & $(+0.2872,-0.0089,+0.1500)$ & $(000000000000000000000010000)^{T}$ \\
\hline 24 & $(+0.2786,-0.0165,+0.2552)$ & $(000000000000000000000001000)^{T}$ \\
\hline 25 & $(+0.2826,-0.0097,+0.1860)$ & $(000000000000000000000000100)^{T}$ \\
\hline 26 & $(+0.2856,-0.0007,+0.1490)$ & $(000000000000000000000000010)^{T}$ \\
\hline 27 & $(+0.2777,-0.0122,+0.2536)$ & $(000000000000000000000000001)^{T}$ \\
\hline
\end{tabular}

Table 1: Eigen value and eigen vector by Kronecker product method

\begin{tabular}{|l|l|l|l|}
\hline Iteration & Initial Gauss & Approx Eigen 3-tuples & $\begin{array}{l}\text { Approx } \\
\text { Eigenvec- } \\
\text { tors }\end{array}$ \\
\hline 3 & $x=y=z=(111)^{T}$ & $(0.1363,-0.0001,0.1939)$ & $\mathrm{u}$ \\
\hline 8 & $\begin{array}{l}x=(123)^{T} ; y=(211)^{T} ; z= \\
(111)^{T}\end{array}$ & $(0.2838,-0.0089,0.1631)$ & $\mathrm{v}$ \\
\hline 6 & $\begin{array}{l}x=(223)^{T} ; y=(256)^{T} ; z= \\
(345)^{T}\end{array}$ & $(0.2785,-0.0149,0.2527)$ & $\mathrm{W}$ \\
\hline
\end{tabular}

Table 2: Approximate eigen value and eigen vectors by gradient method

\section{Numerical Illustrations}

Let us consider the following three-parameter problems with real matrices.

$$
\left(\begin{array}{lll}
1 & 0 & 0 \\
0 & 4 & 0 \\
0 & 0 & 6
\end{array}\right)=\lambda_{1}\left(\begin{array}{ccc}
20 & 0 & 0 \\
0 & 18 & 0 \\
0 & 0 & 20
\end{array}\right)+\lambda_{2}\left(\begin{array}{lll}
1 & 0 & 0 \\
0 & 4 & 0 \\
0 & 0 & 5
\end{array}\right)+\lambda_{3}\left(\begin{array}{lll}
7 & 0 & 0 \\
0 & 8 & 0 \\
0 & 0 & 2
\end{array}\right),
$$




\begin{tabular}{|c|c|}
\hline $\mathrm{u}$ & $\begin{array}{lllllllllll}0.0097 & 0.0064 & -0.0020 & 0.0091 & 0.0060 & -0.0018 & 0.0096 & 0.0063 & -0.0019 & 0.9234 \\
0.6052 & -0.1855 & 0.8688 & 0.5695 & -0.1745 & 0.9129 & 0.5984 & -0.1834 & 0.0092 & 0.0061 & - \\
0.0019 & 0.0087 & 0.0057 & -0.0017 & 0.0091 & 0.0060 & -0.0018)^{T} & & & & \end{array}$ \\
\hline $\mathrm{V}$ & $\begin{array}{llllllllllll}(-0.1068 & -0.1418 & -0.0066 & -0.0530 & -0.0704 & -0.0033 & -0.0528 & -0.0702 & -0.0033 & 0.2583 \\
0.3430 & 0.0160 & 0.1283 & 0.1704 & 0.0080 & 0.1278 & 0.1698 & 0.0079 & 3.5234 & 4.6790 & 0.2184 \\
1.7499 & 2.3238 & 0.1085 & 1.7437 & 2.3156 & 0.1081)^{T} & & & & \end{array}$ \\
\hline $\mathrm{W}$ & $\begin{array}{lllllllllll}(-0.0098 & 0.0081 & -0.0261 & -0.0248 & 0.0206 & -0.0662 & -0.0306 & 0.0254 & -0.0816 & -0.0020 \\
0.0016 & -0.0052 & -0.0050 & 0.0041 & -0.0133 & -0.0061 & 0.0051 & -0.0164 & -0.3240 & 0.2688 & - \\
0.8638 & -0.8219 & 0.6819 & -2.1912 & -1.0133 & 0.8407 & -2.7015)^{T} & & & & \end{array}$ \\
\hline
\end{tabular}

Table 3: Approximate eigenvectors of Table 2

$$
\begin{aligned}
& \left(\begin{array}{lll}
1 & 0 & 0 \\
0 & 1 & 0 \\
0 & 0 & 1
\end{array}\right)=\lambda_{1}\left(\begin{array}{lll}
4 & 0 & 0 \\
0 & 3 & 0 \\
0 & 0 & 2
\end{array}\right)+\lambda_{2}\left(\begin{array}{ccc}
50 & 0 & 0 \\
0 & 52 & 0 \\
0 & 0 & 26
\end{array}\right)+\lambda_{3}\left(\begin{array}{ccc}
2 & 0 & 0 \\
0 & 4 & 0 \\
0 & 0 & 3
\end{array}\right), \\
& \left(\begin{array}{lll}
5 & 0 & 0 \\
0 & 5 & 0 \\
0 & 0 & 6
\end{array}\right)=\lambda_{1}\left(\begin{array}{lll}
2 & 0 & 0 \\
0 & 5 & 0 \\
0 & 0 & 1
\end{array}\right)+\lambda_{2}\left(\begin{array}{lll}
6 & 0 & 0 \\
0 & 4 & 0 \\
0 & 0 & 9
\end{array}\right)+\lambda_{3}\left(\begin{array}{ccc}
24 & 0 & 0 \\
0 & 24 & 0 \\
0 & 0 & 23
\end{array}\right)
\end{aligned}
$$

The exact number of common 3-tuples of the above system will be $3^{3}=27$ as shown in Table 1 calculated by Kronecker Product Method. In table 2 approximate eigenvalues and the corresponding approximate eigenvectors with three different initial guesses has been calculated using Gradient Method.

\section{Conclusion}

The Gradient Method discussed above can be easily extended to $n$-parameter cases in a similar manner. This Method is more efficient for the matrices of higher order than Kroneecker Product Method, where the computation cost as well as time is more due to significantly increase of the dimension of $\Delta_{i}$ of order $N=n^{n}$. Since this Method does not require the inverting matrices as that in Kronecker product Method and hence it can handle large sparse matrices also. The prime benefit of this Method is that no initial approximation is required for eigenvalues. The convergence of this Method is very fast if we choose the initial guess of the eigenvetors near to the exact value. In near future, we have a plan to find the actual order of convergence of the Method for MMEPs.

\section{Acknowledgments}

The authors would like to thank Dr. Ankur Bharali for his support, helpful discussions and careful reading of the manuscript. 


\section{References}

[1] A. R. Curtis, E. K. Blum, A convergent Gradient method for matrix eigenvectoreigentuple problems, Numer. Math., 31 (1978), 247-263, doi: 10.1007/BF01397878.

[2] B Plestenjak, A continuation method for a right definite two-parameter eigenvalue problem, Siam J. Matrix Anal. Appl., 21 (2000), 1163-1184, doi: $10.1137 /$ S0895479898346193.

[3] E. K. Blum, G.H. Rodrigue, Solution of eigenvalue problems in Hilbert spaces by gradient method, J. Comput. System Sci., 8 (1974), 220-237, doi: 10.1016/S0022-0000(74)800565.

[4] F. V. Atkinson, Multiparameter Eigenvalue Problems, Academic Press, New York (1972). ISBN: 0120658011

[5] F. V. Atkinson, Multiparameter spectral theory, Bull. Amer. Math. Soc., 74 (1968), 1-27, http://projecteuclid.org/euclid.bams/1183529377.

[6] M. E. Hochstenbach, B. Plestenjak, A jacobi-davidson type method for a right definite two-parameter eigenvalue problem, Siam J. Matrix Anal. Appl., 24, No. 2 (2002), 392410, doi: 10.1137/S0895479801395264.

[7] P. B. Geltner, E. K Blum, Numerical solution of eigentuple-eigenvector problems in Hilbert spaces by a gradient method, Numer. Math., 31 (1978), 231-246, doi: 10.1007/BF01397877.

[8] T. Kosir, B. Plestenjak, On stability of invariant subspaces of commuting matrices, Linear algebra and its application, 342 (2002), 133-147, doi: 10.1016/S0024-3795(01)00442-6.

[9] Xingzhi Ji, A two-dimensional bisection method for solving two-parameter eigenvalue problems, Siam J. Matrix Anal. Appl., 13, No. 4 (1992), 1085-1093, doi: $10.1137 / 0613065$. 
